\title{
Circulating endothelial cells in patients with heart failure and left ventricular dysfunction ${ }^{1}$
}

\author{
Vicenta Martínez-Sales ${ }^{\mathrm{a}, *}$, Ignacio Sánchez-Lázaro ${ }^{\mathrm{b}, \mathrm{d}}$, Virtudes Vila ${ }^{\mathrm{a}}$, Luis Almenar ${ }^{\mathrm{b}}$, \\ Teresa Contreras ${ }^{\mathrm{c}}$ and Edelmiro Reganon ${ }^{\mathrm{a}}$ \\ ${ }^{a}$ Centro de Investigación, Hospital Universitario La Fe, Valencia, Spain \\ ${ }^{\mathrm{b}}$ Unidad de Insuficiencia Cardiaca y Transplante, Servicio de Cardiología, Hospital Universitario La Fe, Valencia, \\ Spain \\ ${ }^{\mathrm{c}}$ Servicio de Análisis Clínicos, Hospital Universitario La Fe, Valencia, Spain \\ ${ }^{\mathrm{d}}$ Department de Medicina de la Univesitat Autonoma de Barcelona, Barcelona, Spain
}

\begin{abstract}
Introduction and Aims: Acute and chronic heart failure may manifest different degrees of endothelial damage and angiogenesis. Circulating endothelial cells (CEC) have been identified as marker of vascular damage. The aim of our study was to evaluate the evolution of the CEC at different stages of patients with heart failure. We also investigated a potential correlation between CEC and markers of vascular damage and angiogenesis.

Methods: We studied 32 heart failure patients at hospital admission (acute phase) and at revision after 3 months (stable phase) and 32 controls. Circulating markers of endothelial damage (CEC; von Willebrand factor, vWF and soluble E-selectin, sEsel) and angiogenesis (vascular endothelial growth factor, VEGF and thrombospondin-1) were quantified.

Results: Levels of CEC, vWF, sEsel and VEGF are significantly higher in heart failure patients than in controls. Levels of CEC $(36.9 \pm 15.3$ vs. $21.5 \pm 10.0 \mathrm{cells} / \mathrm{ml} ; p<0.001)$, vWF $(325 \pm 101$ vs. $231 \pm 82 \% ; p<0.001)$ and VEGF $(26.3 \pm 15.2$ vs. $21.9 \pm 11.9 \mathrm{ng} / \mathrm{ml} ; p<0.001)$ are significantly higher in the acute phase than in the stable phase of heart failure. CEC levels correlate with vWF and VEGF. Results show than $100 \%$ of patients in acute phase and $37.5 \%$ in stable phase have levels of CEC higher than the 99th percentile of the distribution of controls $(16$ cells $/ \mathrm{ml})$. Therefore, increases in CEC represent a relative risk of 9.5 for heart failure patients suffering from acute phase.

Conclusions: CEC, in addition to being elevated in heart failure, correlate with vWF levels, providing further support for CEC as markers of endothelial damage. Levels of CEC are associated with the acute phase of heart failure and could be used as a marker of the worsening in heart failure.
\end{abstract}

Keywords: Heart failure, circulating endothelial cells, endothelial dysfunction, angiogenesis

\section{Introduction}

Endothelial abnormalities are typical in heart failure $(\mathrm{HF})$ and represent one of the major physiopathological pathways implicated in the development and progression of HF. Circulating endothelial cells (CEC) are a noninvasive marker of vascular damage, remodelling and dysfunction [1-3]. Quantification of

\footnotetext{
* Corresponding author: Dr. V. Martínez-Sales, Centro de Investigación, Hospital Universitario La Fe, Avda. Campanar 21, 46009Valencia, Spain. Tel.: +34 96386 2797; Fax: +34 96197 3018; E-mail: martinez_vicsal@gva.es.

${ }^{1}$ This work is part of the doctoral thesis of Ignacio J. Sánchez Lázaro in the Departament de Medicina (Universitat Autònoma de Barcelona, Spain).
}

CEC in peripheral blood is becoming a novel and reproductive method used to assess endothelial damage/dysfunction [4]. CEC represent mature endothelial cells that have become detached from the endothelial monolayer in response to endothelial injure. Thanks to a consensus definition of CEC and a standardized protocol for identifying these cells [5], good agreement now exists among laboratories with regard to normal CEC counts. Compared with the lower count observed in healthy subjects, increased CEC have been described in a wide spectrum of cardiovascular diseases, such as acute myocardial infarction, unstable angina and congestive $\mathrm{HF}$, in which severe endothelial alterations are implicated [6-8]. Therefore, the increase in CEC reflects severe endothelial damage and the existence of denuded areas of the endothelium. Thus, determina- 
tion of CEC levels can serve as a tool to analyze the process of vascular damage/regeneration in HF.

Current well established markers of endothelial dysfunction are von Willebrand factor (vWF) [9] and soluble E-selectin (sEsel) [10]. Increased values of vWF and sEsel have been documented in $\operatorname{HF}[6,8,11,12]$ and some authors even consider sEsel a prognostic factor in acute HF [13-15].

Concentrations of the angiogenesis markers vascular endothelial growth factor (VEGF) and thrombospondin1 (TSP-1) are increased and decreased, respectively, in chronic heart failure patients, which suggests a role of angiogenesis in the maintenance and repair of luminal endothelium in chronic HF [16]. It has been suggested that high levels of angiogenic factors in heart failure may play a role in the maintenance and repair of a perturbed or damaged endothelium through several different mechanisms [16-18].

The aim of our study was to evaluate the evolution of the CEC at different stages of patients with HF. We also investigated a potential correlation between the number of CEC and the circulating levels of established markers of vascular damage and angiogenesis.

\section{Methods}

\subsection{Study design: Inclusion and exclusion criteria}

Inclusion criteria were to be admitted for heart failure and to agree to complete a follow-up visit three months after discharge. Fifty-one patients consecutively admitted to the Cardiology Department of a reference hospital for HF were recruited. A clinical assessment, laboratory tests, electrocardiogram (ECG), echocardiography and coronary arteriography were performed in all patients. HF was defined according to the European Society of Cardiology Guidelines [14,19]. Patients were discharged with optimal medical therapy for their heart disease according to the European Guidelines for HF $[14,19]$. The follow-up period was three months \pm 5 days. At three months, an outpatient visit including laboratory tests, ECG and echocardiography was performed. Nine patients who had preserved ejection fraction, 3 patients who did not give written consent to participate and 7 patients who died (5 in hospital, 2 at home) before the 3-months follow-up visit at were excluded, as it was not possible to analyze the evolution of the study markers. The total number of patients included in the study after applying the exclusion criteria was 32 .
The control group consisted of 32 healthy subjects, without cardiovascular risk factors, recruited among healthy hospital staff or healthy subjects who visited our hospital for medical checkups, age- and sexmatched to patients. All healthy controls had no history of cardiovascular disease, clinical evidence of vascular, metabolic or inflammatory disease and none was taking prescription medicines. The examination included a clinical examination, ECG and echocardiographic study. The study was conducted in accordance with the principles outlined in the Declaration of Helsinki. The study protocol was approved by the Ethics Committee and all participants gave their informed consent to take part on it.

\subsection{Biomarkers analyzed and blood collection}

Circulating markers of endothelial damage/activity (CEC, vWF and sEsel) and angiogenesis (VEGF and TSP-1) were tested. Venous blood samples were obtained from patients within 24 hours of hospital admission (acute phase) and at a 3-month revision (stable phase). The first 3 milliliters were discarded to avoid CEC damaged by the puncture. Blood for quantification of CEC was collected in a BD Vacutainer tube containing ethylenediaminetetraacetic acid $(1.8 \mathrm{mg} / \mathrm{ml})$ and prepared for immunomagnetic separation within 1 hour. For vWF and sEsel determinations, blood was collected in a BD Vacutainer tube containing sodium citrate $(129 \mathrm{mM})$ at a ratio of $1: 9(\mathrm{v} / \mathrm{v}$, sodium citrate/blood). For VEGF and TSP-1 determinations, blood was collected in a dry BD Vacutainer tube. Samples were centrifuged at $1,500 \mathrm{xg}$ for $30 \mathrm{~min}$ at $4^{\circ} \mathrm{C}$ to obtain plasma or serum and then stored at $-80^{\circ} \mathrm{C}$ to allow later batch analysis.

\subsection{Biomarkers determination}

The isolation and enumeration of CEC was performed by immunomagnetic isolation, following the definition and consensus protocol (5). CEC were isolated from whole blood at $4^{\circ} \mathrm{C}$ with Pan-Mouse M450 Dynabeads (Dynal, Olso, Norway) coated with s-Endo 1 (Biocytex, Marselle, France) a monoclonal antibody raised against the endothelial antigen CD-146. To avoid nonspecific binding of leukocytes to CD-146 coated beads, after immunomagnetic isolation, cells were incubated with lectin, FITC labeled, from Ulex europaeus UEA1 (Sigma-ALDRICH, Inc) for $1 \mathrm{~h}$ in darkness. Thus we confirm the endothelial mature of the isolated cells. Samples were washed, suspended in buffer 
and counted with fluorescence microscopy using a Nageotte camber. A nucleated cell $>10 \mu \mathrm{m}$ in length, with more than five immunomagnetic beads attached and staining UEA-1 positive were regarded as CEC. Conglomerates were counted as one cell. The number of CEC was expressed as cells per milliliter of blood. Reproducibility was tested by performing six replicates of ten different samples. The coefficient of variation was $12 \%$.

VWF antigen level was measured in a hemostatic testing system (ACLTop 700 CTS, Instrumentation Laboratory) using latex particles coated with a polyclonal antibody directed against vWF. The coefficient of variation was $7.5 \%$.

Commercial ELISAs were used to determine sEsel (R\&D Systems) and VEGF (Biosource) levels, showing a coefficient of variation of $6.5 \%$ and $4.9 \%$, respectively.

TSP-1 was determined by indirect ELISA as previously described [20]. The coefficient of variation was $4.8 \%$.

\subsection{Statistical analysis}

Kolmogorov-Smirnov test was used to evaluate whether each parameter came from a normal distribution. Statistical comparisons were performed were performed using the t-test, analysis of variance (ANOVA) with the Bonferroni post-hoc. Bivariate correlation was performed using the Spearman rank correlation test. Measures of association for categorical variables with the Pearson Chi-Square test with Yate's correction was performed to estimate relative risk of independent variables dichotomized as values grater than $99 \%$ confiance interval of CEC (16 cells $/ \mathrm{ml}$ ) and $95 \%$ confidence interval of VWF (174\%), sEsel $(27 \mathrm{ng} / \mathrm{ml})$, VEGF $(245 \mathrm{pg} / \mathrm{ml})$, TSP-1 $(64 \mu \mathrm{g} / \mathrm{ml})$ of distribution in healthy controls. Multiple regression analysis was performed to study the relations among CEC levels and clinical variables (dislipemia, arterial hypertension, diabetes mellitus and smoker) in admission HF patients. Values of $p \leqslant 0.05$ were regarded as statistically significant. All statistical calculations were performed using SPSS $11.5^{\circledR}$ (SPSS Inc., Chicago, Ill, USA) computer software.

\section{Results}

The clinical characteristics, biochemical parameters and treatment of the study group are shown in Table 1,
Table 1

Clinical characteristics, biochemical parameters and treatment of 32 acute heart failure patients and 32 healthy controls

\begin{tabular}{|c|c|c|}
\hline & Acute HF & Healthy controls \\
\hline Age (years) & $68 \pm 10$ & $65 \pm 14$ \\
\hline Sex (Male) & 23 & 22 \\
\hline \multicolumn{3}{|l|}{ Etiology } \\
\hline Ischemic & 9 & NA \\
\hline IDCM & 8 & NA \\
\hline Valvular & 5 & NA \\
\hline Other & 10 & NA \\
\hline Sinus rhythm* & 19 & 32 \\
\hline $\begin{array}{l}\text { Creatinine } \\
(\mathrm{mg} / \mathrm{ml})^{*}\end{array}$ & $1.47 \pm 0.61$ & $0.94 \pm 0.15$ \\
\hline $\begin{array}{l}\text { B-type natriuretic } \\
\text { peptide }(\mathrm{pg} / \mathrm{ml})^{*}\end{array}$ & $376 \pm 297$ & $7.7 \pm 4.8$ \\
\hline $\mathrm{EF}<40 \% *$ & 32 & 0 \\
\hline $\begin{array}{l}\text { Diabetes } \\
\text { mellitus* }\end{array}$ & 9 & 0 \\
\hline $\mathrm{HT}^{*}$ & 21 & 0 \\
\hline Dyslipidemia* & 15 & 0 \\
\hline \multicolumn{3}{|l|}{ Smoker* } \\
\hline Yes & 4 & 2 \\
\hline No & 14 & 4 \\
\hline Ex-smoker & 10 & 6 \\
\hline BMI $\left(\mathrm{kg} / \mathrm{m}^{2}\right)^{*}$ & $27 \pm 5$ & $24 \pm 2$ \\
\hline Aspirin & 11 & NA \\
\hline Clopidogrel & 4 & NA \\
\hline Acenocoumarol & 12 & NA \\
\hline Beta-blocker & 16 & NA \\
\hline ACEI & 15 & NA \\
\hline AIIRA & 4 & NA \\
\hline Statins & 12 & NA \\
\hline Diuretics & 28 & NA \\
\hline Antialdosterones & 15 & NA \\
\hline Digoxin & 5 & NA \\
\hline Amiodarone & 3 & NA \\
\hline \multicolumn{3}{|c|}{$\begin{array}{l}\text { Data are expressed as mean } \pm \text { SD or as number of pa- } \\
\text { tients unless stated. }{ }^{*} p<0.05 \text {. ACEI: Angiotensin- } \\
\text { converting enzyme inhibitors; AIIRA: Angiotensin II re- } \\
\text { ceptor antagonists; BMI: body mass index; BNP: B-type } \\
\text { natriuretic peptide; EF: Ejection fraction; HF: Heart fail- } \\
\text { ure; HT: Systemic hypertension; IDCM: Idiopathic di- } \\
\text { lated cardiomyopathy; NA: Not applicable. }\end{array}$} \\
\hline
\end{tabular}

and levels of CEC and other analyzed markers, both in acute and stable phases and in controls, are shown in Table 2. Patient's hospitalization was always caused by HF in any of their chances (volume overload, pulmonary edema, dyspnea with minimal effort) and never by ischemic or arrhythmic causes. Patients were treated according to clinical practice guidelines. There were no changes in the patient's treatments for discharge to the three months follow-up. When comparing the levels of CEC, vWF, sEsel, TSP-1 and VEGF, among the smoker/non-smoker groups of acute HF patients, no significant differences were observed. A multiple regression analysis was performed in order to asses the influence of diabetes, hypertension and dislipemia in 
Table 2

Circulating levels of endothelial dysfunction and angiogenesis markers in 32 heart failure patients determined at acute phase (hospital admission) and stable phase (after 3 months) and in 32 healthy controls

\begin{tabular}{lcccccc}
\hline & Acute HF & Stable HF & Controls & $\mathrm{p} 1$ & $\mathrm{p} 2$ & $\mathrm{p} 3$ \\
\hline Endothelial markers & & & & & & \\
CECs (cells/ml) & $36.9 \pm 15.3$ & $21.5 \pm 10.0$ & $8.6 \pm 5.2$ & $<0.001$ & $<0.001$ & $<0.001$ \\
vWF:Ag $(\%)$ & $325 \pm 101$ & $231 \pm 82$ & $122 \pm 27$ & $<0.001$ & $<0.001$ & $<0.001$ \\
sEsel $(\mathrm{ng} / \mathrm{ml})$ & $26.3 \pm 15.2$ & $21.9 \pm 11.9$ & $17.1 \pm 6.4$ & 0.3 & $<0.01$ & $<0.05$ \\
Angiogenesis markers & & & & & & \\
VEGF $(\mathrm{pg} / \mathrm{ml})$ & $411 \pm 312$ & $259 \pm 226$ & $143 \pm 64$ & $<0.05$ & $<0.001$ & $<0.05$ \\
TSP-1 $(\mu \mathrm{g} / \mathrm{ml})$ & $57.8 \pm 19.1$ & $49.4 \pm 15.3$ & $47.1 \pm 9.6$ & 0.5 & $<0.05$ & 0.8 \\
\hline
\end{tabular}

Results are expressed as mean \pm SD. CECs: circulating endothelial cells; HF: heart fauilure; sEsel: soluble E-selectin; TSP-1: thrombospondin-1; VEGF: vascular endothelial growth factor; vWF: von Willebrand factor; p1: Acute HF vs. Stable HF; p2: Acute HF vs. Control; p3: Stable HF vs. Control.

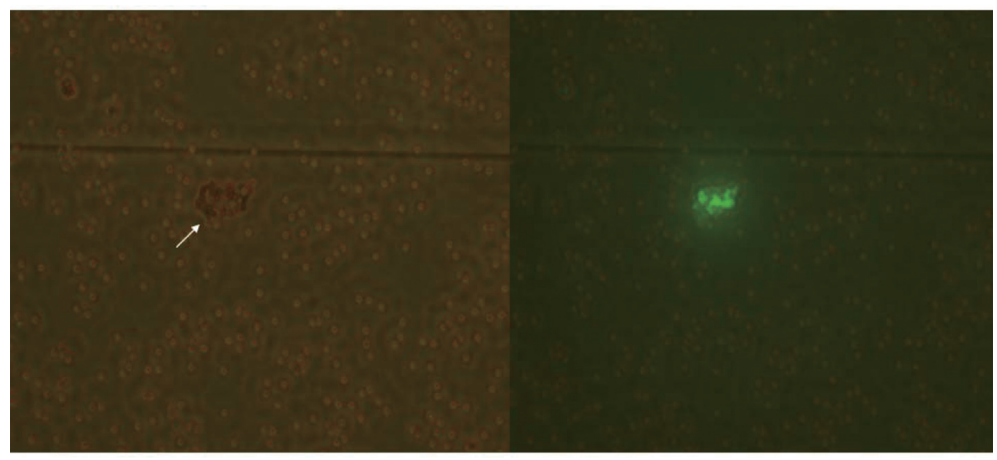

Fig. 1. A circulating endothelial cell, forming a rosette of $10 \mu \mathrm{m}$ in length with more than six immunomagnetic beads (left), and staining bright green with Ulex Europaeus lectin (right). Photomicrograph in epifluorescence (Leica), wet preparation. For scale, the large numbers of residual immunomagnetic beads each have a diameter of $4.5 \mu \mathrm{m}$.

CEC level in admission HF patients. The results show no statistically significant correlation between these variables (coefficient of multiple correlations: 0.33 , signification: 0.69 and a coefficient of determination: 0.106).

Figure 1 shows a typical, single CEC from a patient with HF; CD146-based immunomagnetic isolation with subsequent UEA-1 stain. None stained for the leukocyte-common antigen CD45. The distribution of the values of CEC in the three study groups is shown in Fig. 2. For HF patients, median and (interquartile range) CEC levels were 35 (25-43) cells/ml at hospital admission and 20 (16-22) cells/ml at stable phase, while healthy controls showed 6 (4-12) cells/ml. CEC counts in peripheral blood were significantly higher in HF patients both at acute and at stable phase than in controls $(p<0.001)$.

Table 2 summarizes the mean of circulating levels of endothelial dysfunction and angiogenesis markers determined in patients with acute and stable HF and in controls. Patients showed higher vWF levels in both at acute $(p<0.001)$ and at the stable phase $(p<0.001)$ than in controls. When comparing the two phases of
HF patients, significantly higher levels of vWF were found in patients at acute phase $(p<0.001)$. sEsel levels were significantly increased in the patients, at acute phase $(p<0.01)$ as well as at stable phase $(p<$ 0.05 ) when compared to healthy controls. VEGF levels were raised in patients at acute phase compared to the levels at the stable phase $(p<0.05)$. Moreover, VEGF levels were significantly increased in acute and stable HF ( $p<0.001$ and $p<0.05$, respectively) as compared with controls. Levels of TSP-1 increased significantly in patients, only in the acute phase $(p<0.05)$ (Table 2$)$.

Figure 3 shows temporal changes analysis in levels of CEC, sEsel, vWF, VEGF and TSP-1 in HF patients. Results represent the percentage of patients with CEC levels higher than the 99th percentile of the distribution of healthy controls as the top cutoff point of the normal range (CEC level above 16 cells/ml) and levels higher than the 95th percentile for vWF (level above $174 \%$ ), sEse (level above $27 \mathrm{ng} / \mathrm{ml}$ ), VEGF (level above $245 \mathrm{pg} / \mathrm{ml}$ ) and TSP-1 (level above $64 \mu \mathrm{g} / \mathrm{ml}$ ).

The study of the association of patient groups with levels of CEC dichotomized as greater than 16 cells/, shows that all acute HF patients $(100 \%)$ have high num- 


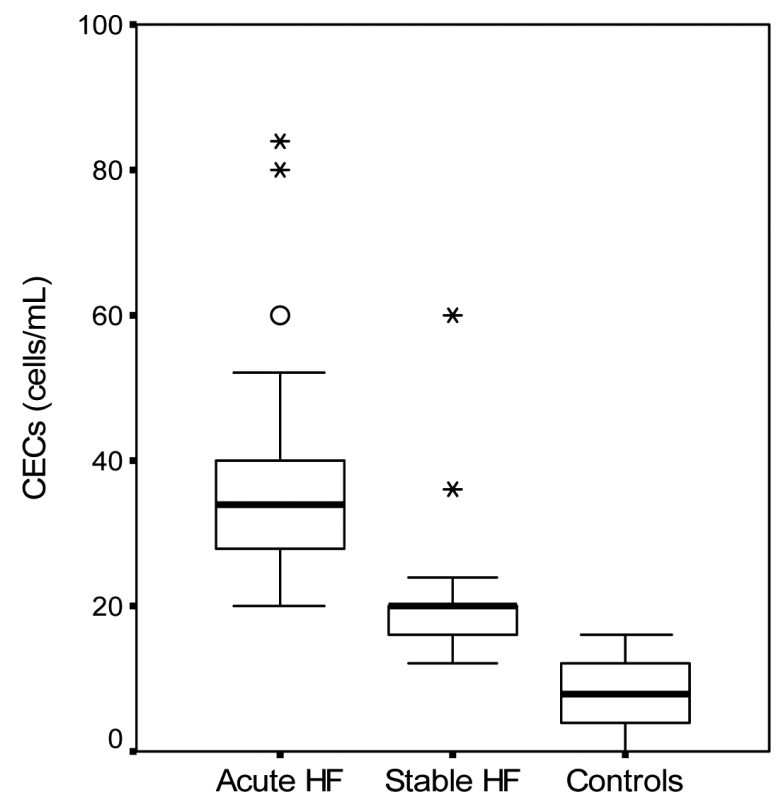

Fig. 2. Distribution of circulating endothelial cells in 32 heart failure patients at acute (hospital admission) and at stable phase (3-month revision) and in 32 healthy controls. Bloxplot showing median (line), interquartile range (boxes), $5 \%$ to $95 \%$ percentile (whiskers), and outliers (dot) and extremes (stars) cell levels. CECs: Circulating endothelial cells; HF: Heart failure.

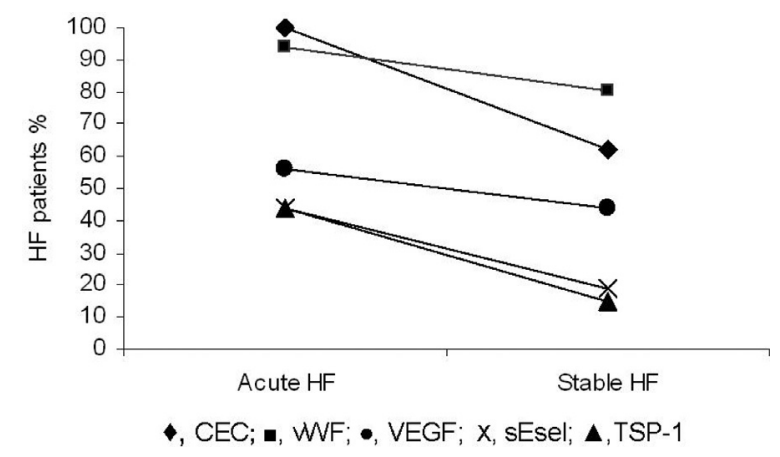

Fig. 3. Circulating endothelial cells, soluble E-selectin, von Willebrand factor, vascular endothelial growth factor and thrombospondin-1 levels at acute and at stable phase in HF patients. Data represent the percentage of patients with marker levels higher than the $99 \%$ confidence interval of distribution in healthy controls (CECs) and $95 \%$ confidence interval of distribution in healthy controls (sEsel, TSP-1, VEGF and vWF). CECs: Circulating endothelial cells; HF: Heart failure; sEsel: Soluble E-selectin; TSP-1: Thrombospondin-1; VEGF: vascular endothelial growth factor; vWF: von Willebrand factor.

ber of CEC (Chi-square $=32.20, p<0.0001$ and a relative risk $=3.625,95 \%$ confidence interval: 2.01 to 6.54$)$ and 12 of 32 patients $(37,5 \%)$ in stable group (Chi-square $=23.01, p<0.0001$ and a relative risk $=$ $0.38,95 \%$ confidence interval: 0.22 to 0.66 ). By nor- malizing the value of the RR in the acute to the value in patients with stable HF, it has been possible to establish that the risk of having the highest number of CEC in the acute phase is 9.5 times higher than in the stable phase of the disease.

The cutoff point of the normal range ( 95 th percentile) was exceeded by a large number of acute heart failure (vWF: 30 patients) and stable HF patients (vWF: 26 patients), while levels of sEsel (acute: 14 patients; stable HF: 5 patients), VEGF (acute: 18 patients; stable HF: 14 patients) and TSP-1 (acute: 14 patients; stable HF: 6 patients) were exceeded only by a small number of patients. The probability of heart failure patients having sEsel, vWF, VEGF and TSP-1 levels above the cutoff point against controls was significant for $\mathrm{vWF}$ $(p<0.001, \mathrm{RR}=5.94 ; 95 \%$ Confidence Interval: 2.84 to 12.4 ), and VEGF ( $p<0.01$, relative risk $=2.33$; 95\% confidence Interval: 1.55 to 3.51 ).

In the whole study group, CEC correlated well with vWF (Spearman $r=0.678, p<0.001$ ) and modestly with VEGF $(r=0.39, p<0.01)$. Levels of vWF and TSP-1 were modestly correlated $(r=0.269, p=0.05)$.

\section{Discussion}

The results obtained in this study show that patients admitted for acute HF have elevated CEC levels that decrease 3-month after hospital discharge, but without reaching the values of healthy subjects. CEC are a novel marker of endothelial damage, and their concentration correlates with other markers of endothelial function (vWF) and angiogenesis (VEGF). These data reflect a continued endothelial activation through endothelial damage and endothelial maintenance and repair of luminal endothelium.

Patient recruitment as well as sample collection was done within the first 24 hours of hospital admission, to coincide with the acute phase of disease [13]. Evaluation at three months after discharge was done arbitrarily. since neither the clinical practice guidelines nor the scientific literature establish a temporal criterion to distinguish between the two phases of HF [13,15,21,22]. Levels in our study are also higher than those published in HF patients without hospital admissions for at least six months, so we think that more than three months are needed to stabilize the inflammatory state [16,23]. This study is consistent with previous observations on raised CEC in patients with acute and/or stable $\mathrm{HF}$ as compared with healthy controls [6,8]. Our study also showed that acute HF was associated with high 
numbers of CEC, which significantly decreased after 3 months of treatment. This decrease was associated with the clinical improvement of the studied patients. These results do not coincide with those of Chong et al. [6], who reported that number of CEC increased in both patients groups, but with no significant difference between them. This discrepancy could be due to the fact that our patients enter the study in the acute phase and follow-up has been performed until their stabilization; while Chong et al. [6] studied two different patient groups, one with acute HF and other with chronic HF. In addition, highest levels of CEC in our study may differ from those of Chong et al. [6] because of the different techniques used to quantify CEC.

CEC are cells from the vascular endothelium that have become detached in response to damage. As shown by Makin et al. [7] in acute myocardial infarction and Chong et al. [6] in HF, our study revealed that $\mathrm{CEC}$, in addition to being elevated in HF, were positively correlated with vWF levels. This latter finding provides further support for CEC as early markers of endothelial damage $[6,13,24]$. CEC reflect an impairment in vasodilation produced by an endothelial denudation in HF, while in pulmonary hypertension the CEC levels reflect an endothelial proliferation. Recent studies have shown that the effect of clopidogrel after a stent placement could decrease the levels of CEC [25]. In our study only few patients were treated with clopidogrel, so we think that this fact didn't affect our results. As an addition to the studies conducted in HF, our study is the first to draw these conclusions in the same population of patients at different phases of the disease.

Studies have found that vWF is a suitable marker of endothelial dysfunction, increased levels being found in inflammatory and atherosclerotic vascular diseases [9]. $\mathrm{HF}$ is associated with impaired endothelium, which contributes to the peripheral vasoconstriction that is characteristic of HF [17], and high levels of vWF are associated with HF $[6,8,16,26,27]$. Our data showed that CEC were strongly correlated with vWF $(p<$ 0.001 ) so the correlation between CEC and vWF reveals the presence of endothelial damage in these patients.

Although more weakly, E-selectin [12] is elevated in HF patients and some authors even consider it a prognostic factor in acute heart failure [13-15]. Eselectin promotes adherence of leukocytes to vascular endothelium, facilitates its permeability and acts as a chemotactic factor. Consequently, it plays a key role in maintenance of inflammation in HF. This role may be modified by drugs such as statins [28], which may explain why the differences between the two phases of HF in our study were smaller than with other markers.

Analysis of the angiogenesis parameter in our patients showed that HF leads to progression of the angiogenesis response with high levels of VEGF. High levels of angiogenic factors in HF may play a role in the maintenance and repair of a damaged endothelium through different mechanisms [16-18]. VEGF is one the growth factors implicated in angiogenesis. The myocardial ischemia occurring in HF promotes the release of VEGF, especially in phases of decompensation. In spite of this, the role of VEGF in HF is unclear; as there is insufficient evidence that true angiogenesis occurs in HF. Thus, elevated VEGF levels may only reflect the process of vascular repair, which would also explain the biphasic nature (raised in acute heart failure but diminished in very late phases of the disease) observed in some studies $[16,17,29]$. Our results would support the theory that VEGF is increased as a result of the need for endothelial repair, since we observed a significant correlation with $\mathrm{CEC}$, which would indicate the presence of endothelial damage.

Regarding the study limitations and despite the fact that the sample enabled us to draw novel and important conclusions, a larger number of patients would have allowed us to explore other relationships, such as the possible influence of drugs the different markers and different etiologies. Another limitation is the potential effect of oral anticoagulant treatment on the study parameters. When comparing our data with those of other studies, it should not be forgotten that our hospital is a tertiary care facility. This means that patients diagnosed with $\mathrm{HF}$ are received by other departments in the hospital (Short Stay Unit, Internal Medicine), and only those with more severe illness (from which the study patients were recruited) are referred to the Cardiology Department.

\section{Conclusions}

Our study revealed that CEC, in addition to being elevated in $\mathrm{HF}$, were positively correlated with vWF levels, providing further support for CEC as marker of endothelial damage. Levels of CEC are associated with the acute phase of HF patients. This way the levels of CEC could be use as a marker of worsening in $\mathrm{HF}$, although these conclusions should be confirmed by larger studies.

Finally, we want to point out that this study is the first to draw these conclusions in the same population of patients at different phases of the disease. 


\section{Acknowledgments}

The authors wish to thank Josefa Llorés Alegre, Ursula Salinas and Daniel Hernandez for their expert technical assistance.

We also thank Ministerio de Ciencia e Innovación, Instituto de Salud Carlos III (RETICS: REDINSCOR grant RD06/0003/1001, RECAVA grant RD06/0014/ 0004 and RENEVAS grant RD06/0026/0006).

This study is part of the doctoral thesis of Ignacio Sánchez-Lázaro in the Universitat Autònoma de Barcelona (Departament de Medicina Interna).

This work was supported in part by research grants from Ministerio de Ciencia, Tecnología e Innovación, Instituto de Salud Carlos III, FIS PI08124; Generalitat Valenciana, Conselleria Sanitat, AP037/07 and Sección de Insuficiencia Cardíaca y Trasplante de la Sociedad Española de Cardiología, 2009.

\section{References}

[1] J. Quilici, N. Banzet, P. Paule, J.B. Meynard, M. Mutin, J.L. Bonnet et al., Circulating endothelial cells count as a diagnostic marker for non-ST-elevation acute coronary syndromes, Circulation 110 (2004), 1586-1591.

[2] A. Widman, F. Sabatier, L. Arnaud, L. Bonello, G. AlMassarani, F. Paganelli et al., CD146-based immunomagnetic enrichment followed by multiparameter flow cytometry: a new approach to counting circulating endothelial cells, J Thromb Haemost 6 (2008), 869-876.

[3] P. Mancuso, A. Burlini, G. Pruneri, A. Goldhirsch, G. Martinelli and F. Bertolini, Resting and activated endothelial cells are increased in the peripheral blood of cancer patients, Blood 97 (2001), 3658-3661.

[4] A.D. Blann, A. Woywodt, F. Bertolini, T.M. Bull, J.P. Buyon, R.M. Clancy et al., Circulating endothelial cells. Biomarkers of vascular diseases, Thromb Haemost 93 (2005), 228-235.

[5] A. Woywodt, A.D. Blann, T. Kirsch, U. Erdbruegger, N. Banzet, M. Haubitz et al., Isolation and enumeration of circulating endothelial cells by immunomagnetic isolation: proposal of a definition and a consensus protocol, J Thromb Haemost 4 (2006), 671-677.

[6] A.Y. Chong, G.Y. Lip, B. Freestone and A.D. Blann, Increased circulating endothelial cells in acute heart failure: Comparison with von Willebrand factor and soluble E-selectin, Eur J Heart Fail 8 (2006), 167-172.

[7] A.J. Makin, A.D. Blann, N.A. Chung, S.H. Silverman and G.Y. Lip, Assessment of endothelial damage in atherosclerotic vascular disease by quantification of circulating endothelial cells. Relationship with von Willebrand factor and tissue factor, Eur Heart J 25 (2004), 371-376.

[8] A.Y. Chong, A.D. Blann, J. Patel, B. Freestone, E. Hughes and G.Y. Lip, Endothelial dysfunction and damage in congestive heart failure: relation of flow-mediated dilation to circulating endothelial cells, plasma indexes of endothelial damage, and brain natriuretic peptide, Circulation 110 (2004), 1794-1798.
[9] A.D. Blann, Plasma von Willebrand factor, thrombosis and the endothelium: the first 30 years, Thromb Haemost 95 (2006), 49-55.

[10] B. Freestone, A.Y. Chong, Blann and G.Y. Lip, The effects of direct current cardioversion for persistent atrial fibrillation on indices of endothelial damage/dysfunction, Thrombosis Res 118 (2006), 479-485.

[11] V. Vila, V. Martinez-Sales, L. Almenar, I. Sánchez Lázaro, P. Villa and E. Reganon, Effect of oral anticoagulant therapy on thrombospondin-1 and von Willebrand factor in patients with stable heart failure, Thromb Res 121 (2008), 611-615.

[12] A.D. Blann and A. Pretorius, Circulating endothelial cells and endothelial progenitor cells: two sides of the same coin, or two different coins? Atherosclerosis 188 (2006), 12-18.

[13] A.Y. Chong, B. Freestone, J. Patel, H.S. Lim, E. Hughes, A.D. Blann et al., Endothelial activation, dysfunction, and damage in congestive heart failure and the relation to brain natriuretic peptide and outcomes, Am J Cardiol 97 (2006), 671-675.

[14] European Society of Cardiology, Heart Failure Association of the ESC (HFA), European Society of Intensive Care Medicine (ESICM), K. Dickstein, A. Cohen-Solal, G. Filippatos, J.J. McMurray, P. Ponikowski, P.A. Poole-Wilson et al., ESC guidelines for the diagnosis and treatment of acute and chronic heart failure 2008: the Task Force for the diagnosis and treatment of acute and chronic heart failure 2008 of the European Society of Cardiology. Developed in collaboration with the Heart Failure Association of the ESC (HFA) and endorsed by the European Society of Intensive Care Medicine (ESICM), Eur J Heart Fail 10 (2008), 933-989.

[15] C. Gürgün, M. Ildizli, O. Yavuzgil, A. Sin, A. Apaydin, C. Cinar et al., The effects of short term statin treatment on left ventricular function and inflammatory markers in patients with chronic heart failure, Int J Cardiol 123 (2008), 102-107.

[16] V. Vila, V. Martínez-Sales, L. Almenar, I. Sánchez Lázaro, P. Villa and E. Reganon, Inflammation, endothelial dysfunction and angiogenesis markers in chronic heart failure patients, Int J Cardiol 130 (2008), 276-277.

[17] G.Y. Lip and I. Chung, Vascular endothelial growth factor and angiogenesis in heart failure, J Card Fail 11 (2005), 285-287.

[18] M. Hristov and C. Weber, Ambivalence of progenitor cells in vascular repair and plaque stability, Curr Opin Lipidol 19 (2008), 491-497.

[19] Task Force for Diagnosis and Treatment of Acute and Chronic Heart Failure 2008 of European Society of Cardiology, K. Dickstein, A. Cohen-Solal, G. Filippatos, J.J. McMurray, P. Ponikowski, P.A. Poole-Wilson et al., ESC Guidelines for the diagnosis and treatment of acute and chronic heart failure 2008: the Task Force for the Diagnosis and Treatment of Acute and Chronic Heart Failure 2008 of the European Society of Cardiology. Developed in collaboration with the Heart Failure Association of the ESC (HFA) and endorsed by the European Society of Intensive Care Medicine (ESICM), Eur Heart $J \mathbf{2 9}$ (2008), 2388-442.

[20] V. Martínez-Sales, V. Vila, M. Ferrando and E. Reganon, Atorvastatin neutralizes the up regulation of thrombospondin-1 induced by thrombin in human umbilical vein endothelial cells, Endothelium 14 (2007), 233-238.

[21] European Society of Cardiology; Heart Failure Association of the ESC (HFA), European Society of Intensive Care Medicine (ESICM), K. Dickstein, A. Cohen-Solal, G. Filippatos, J.J. McMurray, P. Ponikowski, P.A. Poole-Wilson et al., ESC Guidelines for the diagnosis and treatment of acute and chronic heart failure 2008, Eur Heart J 29 (2008), 2388-2442. 
[22] G. Balconi, R. Lehmann, F. Fiordaliso, B. Assmus, S. Dimmeler, P. Sarto et al., Levels of circulating pro-angiogenic cells predict cardiovascular outcomes in patients with chronic Heart Failure, J Card Fail 15 (2009), 747-755.

[23] I.J. Sánchez-Lázaro, L. Almenar, E. Reganon, V. Vila, L. Martínez-Dolz, V. Martínez-Sales et al., Inflammatory markers in stable heart failure and their relationship with functional class, Int J Cardiol 129 (2008), 388-393.

[24] U. Erdbruegger, M. Haubitz and A. Woywodt, Circulating endothelial cells: a novel marker of endothelial damage, Clin Chim Acta 373 (2006), 17-26.

[25] L. Bonello, K. Harhouri, F. Sabatier, L. Camoin-Jau, L. Arnaud, K. Baumstarck-Barrau et al., Level of Adenosine Diphosphate Receptor P2Y12 Blockade During Percutaneous Coronary Intervention Predicts the Extent of Endothelial Injury, Assessed by Circulating Endothelial Cell Measurement J Am Coll Cardiol 56 (2010), 1024-1031.
[26] U.M. Vischer, Von Willebrand factor, endothelial dysfunction, and cardiovascular disease, J Thromb Haemost 4 (2006), 1186-1193.

[27] L. Martínez-Dolz, L. Almenar, E. Reganon, V. Vila, C. Chamorro, L. Andrés et al., Follow-up study on the utility of von Willebrand factor levels in the diagnosis of cardiac allograft vasculopathy, J Heart Lung Transplant 27 (2008), 760-766.

[28] K.A. Eccles, H. Sowden, K.E. Porter, S.M. Parkin, S. HomerVanniasinkam and A.M. Graham, Simvastatin alters human endothelial cell adhesion molecule expression and inhibits leukocyte adhesion under flow, Atherosclerosis 200 (2008), 69-79.

[29] M. Valgimigli, G.M. Rigolin, A. Fucili, M.D. Porta, O. Soukhomovskaia, P. Malagutti et al., CD34_ and endothelial progenitor cells in patients with various degrees of congestive heart failure, Circulation 110 (2004), 1209-1212. 


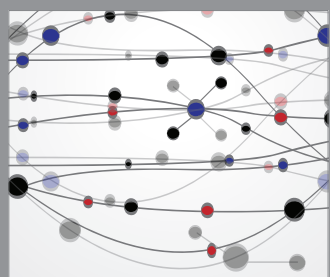

The Scientific World Journal
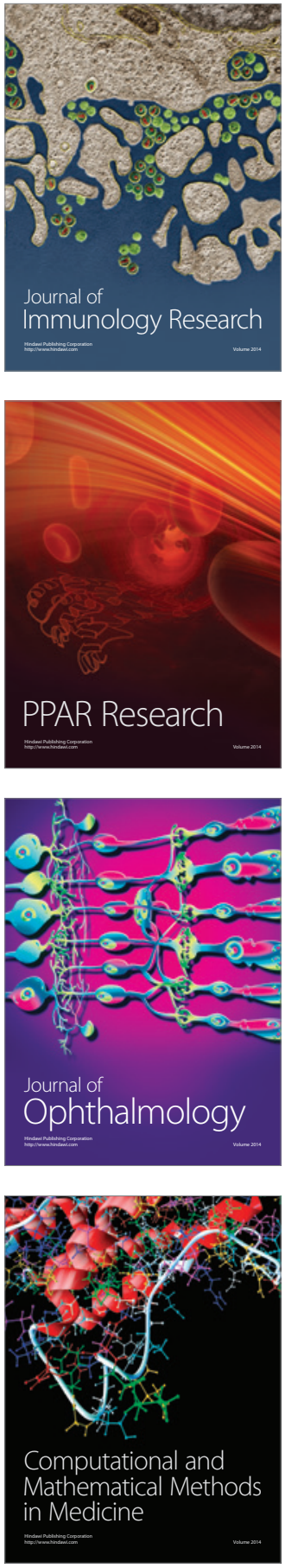

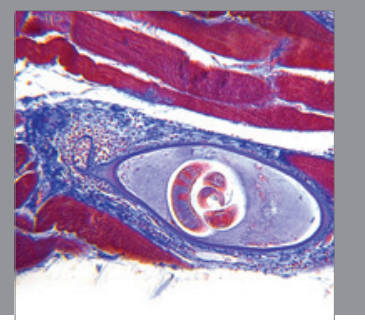

Gastroenterology

Research and Practice
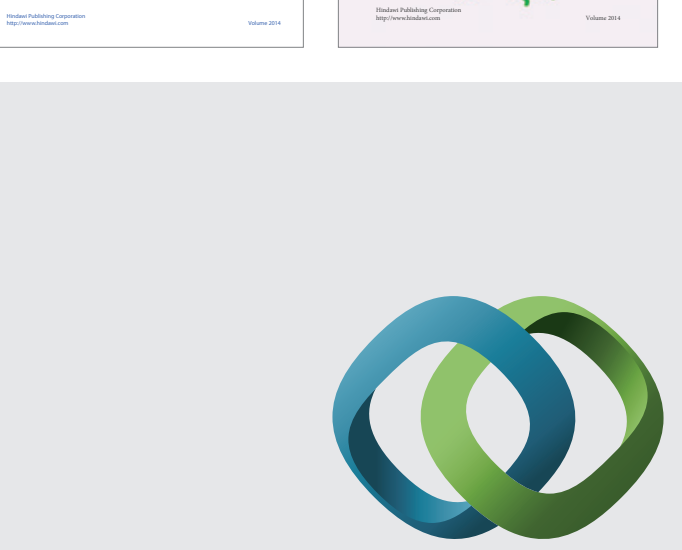

\section{Hindawi}

Submit your manuscripts at

http://www.hindawi.com
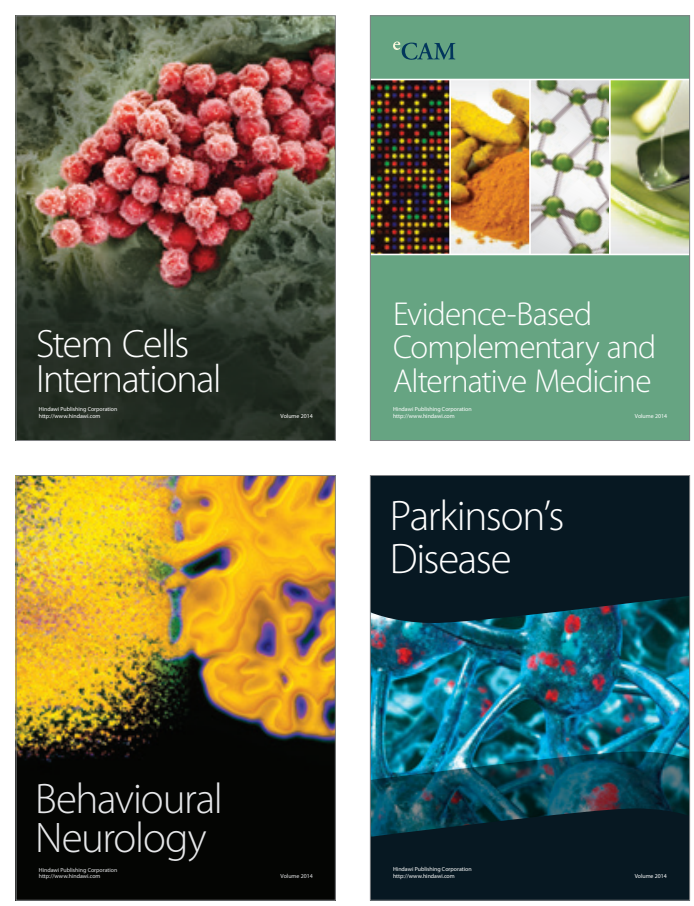

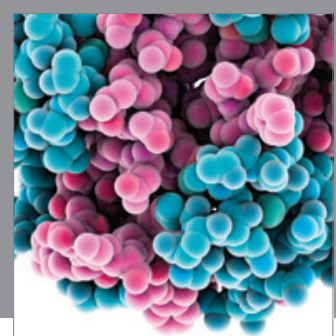

Journal of
Diabetes Research

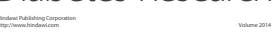

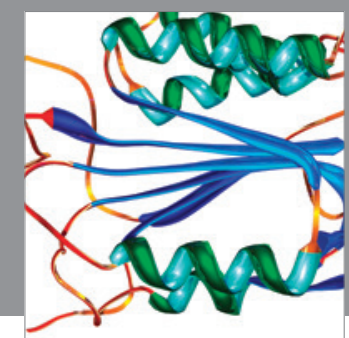

Disease Markers
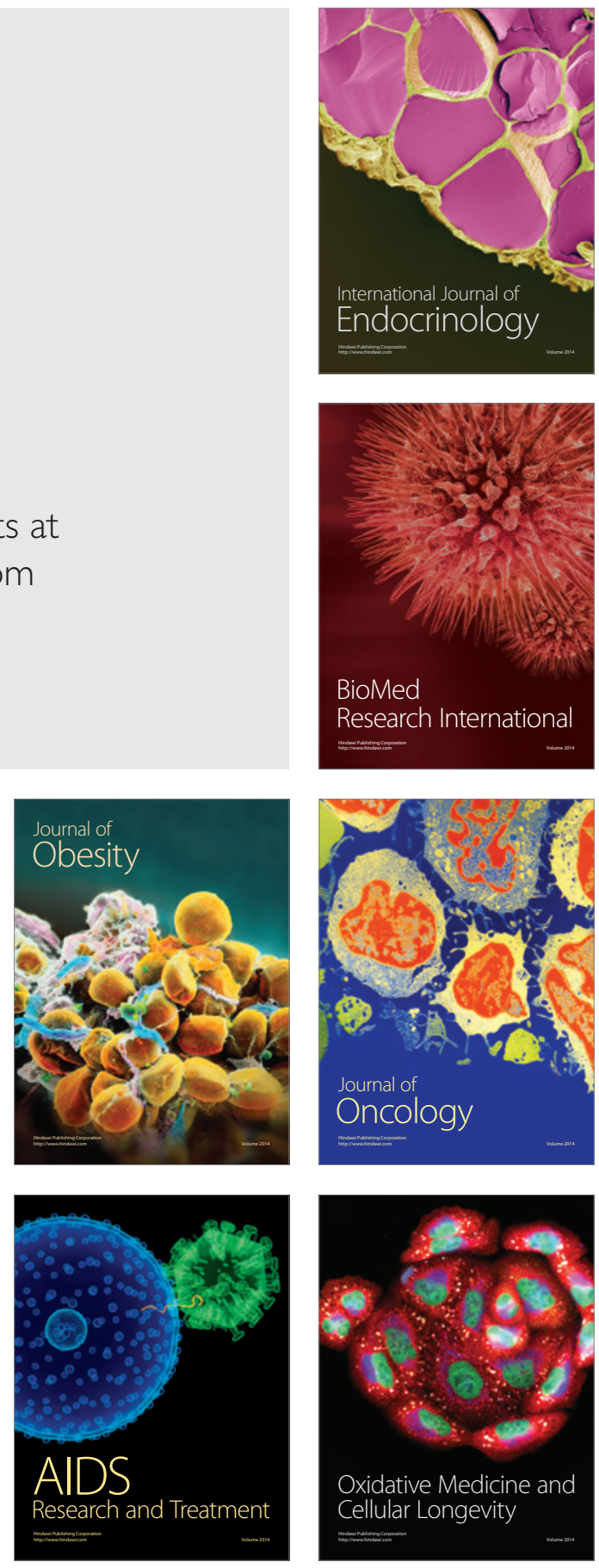